\title{
Influence of Dentin Priming with Tannin-Rich Plant Extracts on the Longevity of Bonded Composite Restorations
}

\author{
Mackeler Ramos Polassi, ${ }^{1}$ Thales de Sá Oliveira, ${ }^{1}$ Ana Calheiros de Carvalho, ${ }^{2}$ \\ Lívia Soman de Medeiros Medeiros $\mathbb{D},{ }^{2}$ Thiago André Moura Veiga $\mathbb{D},{ }^{2}$ \\ Carlos Frederico de Oliveira Graeff $\left(\mathbb{D},{ }^{3}\right.$ Alejandra Hortencia Miranda González, ${ }^{3,4}$ \\ Maria Cristina Marcucci ${ }^{D},{ }^{5}$ Simone dos Santos Grecco ${ }^{(D)}{ }^{4}$ \\ and Paulo Henrique Perlatti D'Alpino (iD ${ }^{3,4}$
}

${ }^{1}$ Universidade Anhanguera de São Paulo, São Paulo, SP, Brazil

${ }^{2}$ Department of Chemistry, Universidade Federal de São Paulo, Diadema, SP, Brazil

${ }^{3}$ São Paulo State University (UNESP), School of Sciences, POSMAT-Post-Graduate Program in Materials Science and Technology, Bauru, SP, Brazil

${ }^{4}$ Triplet Biotechnology Solutions, São Paulo, SP, Brazil

${ }^{5}$ Science and Technology Institute, Universidade Estadual Paulista-UNESP, São José dos Campos, SP, Brazil

Correspondence should be addressed to Paulo Henrique Perlatti D’Alpino; paulodalpino@gmail.com

Received 26 April 2021; Accepted 6 June 2021; Published 16 June 2021

Academic Editor: Piotr Minkiewicz

Copyright (C) 2021 Mackeler Ramos Polassi et al. This is an open access article distributed under the Creative Commons Attribution License, which permits unrestricted use, distribution, and reproduction in any medium, provided the original work is properly cited.

\begin{abstract}
Objective. This in vitro study evaluated the influence of bioactive plant extracts as dentin biomodifying agents to improve the longevity of bonded restorations. For that, plant extracts were applied to the dentin surface prior to the adhesive system. Materials and Methods. Bovine incisors were ground flat to obtain $2 \mathrm{~mm}$ thick slices in which conical preparations were made $(N=10)$. Tannin-containing plant extracts were applied to dentin before the application of the restorative system, as follows: control group (untreated, CTL), chlorhexidine 0.12\% (CHX), mastruz (Dysphania ambrosioides, MTZ), cat's claw (Uncaria tomentosa, CTC), guarana (Paullinia cupana, GUA), galla chinensis (Rhus chinensis, GCH), and tannic acid (extracted from Acacia decurrens, TNA). The push-out bond strength test was conducted $(0.5 \mathrm{~mm} / \mathrm{min})$. Dentin biomodification was assessed by the modulus of elasticity and mass change in bovine tooth sections $(0.5 \times 1.7 \times 7.0 \mathrm{~mm})$. The dentin staining after extract treatments of dentin slices was compared. The dentin surface wettability was also evaluated by means of the contact angles of the adhesive system with the dentin surface and compared with the untreated control group. Data were subjected to ANOVA/Tukey's test $(\alpha=0.05)$. Results. The bond strength of the restoratives to dentin was not significantly improved by the plant extracts, irrespective of the evaluation time $(p>0.05)$. Except for TNA, the elastic modulus of demineralized dentin significantly reduced after treatment with the plant extracts $(p<0.05)$. The dentin staining correlated with the tannin content of the extracts. The contact angle was significantly reduced when treated with CTC, GCH, and TNA. Conclusions. The tannin-containing extracts had a questionable effect on the longevity of bonded restorations. The dentin modulus was negatively affected by the extract treatments. Although some of the extracts changed the contact angle, which seems to improve the adhesive monomer permeation, the tannin-rich plant extract application prior to adhesive application was proven to be clinically unfeasible due to dentin staining.
\end{abstract}

\section{Introduction}

At the tooth-restoration interface occurs an interaction of polymers, collagen, noncollagenous protein, and minerals with the dentin, forming a hybrid layer [1]. The main goal in the bonding of adhesive restorations to dentin is to obtain complete infiltration of the resin monomers into the previously demineralized dentin surface to avoid the degradation of this interfacial area [2]. In this manner, the extent to which the adhesive resin envelops collagen fibrils helps 
improve the durability of bonding to dentin [3]. Exposed unprotected collagen fibrils after the bonding procedure is regarded to be the main problem that impacts the restoration longevity due to the deterioration that occurs at the hybrid layer [4]. As a consequence, there is a decrease in the bond strength of resin-based materials to dentin, a decrease in the sealing of the restoration margins, and ultimately restoration loss [5].

Different approaches have been proposed to enhance and reinforce the dentin by superficially altering its biochemistry and biomechanical properties [6]. Among these purposes, dentin biomodification is claimed to be a biomimetic method mediated by bioactive agents, which interact with various extracellular components of the dentin matrix, ultimately improving the biomechanics and stability of the dentin collagen matrix [6]. In the process of dentin biomodification, bioactive chemical mediators are regarded to improve the strength of collagen fibrils by forming crosslinks [6]. The collagen cross-linking favors the increase of the fibrillar resistance to enzymatic degradation [7] and also provides improved tensile properties [8]. In this manner, synthetic and natural compounds have been proposed to induce collagen cross-linking [9]. Cross-linking is also regarded to maintain the stability of dentin collagen to enzymatic degradation [4]. Inhibitors of matrix metalloproteinases (MMPs) and cysteine cathepsins, which are intensively activated in mildly acidic environments, have been also applied as dentin primers [10, 11]. Tannins, which are polyphenols found in plants, are regarded to inhibit proteases and to present an affinity to dentin collagen, acting as a cross-linking inductor, preventing dentin matrix loss $[6,12]$.

The present in vitro study evaluated the effects of tanninrich plant extracts on the mechanical properties and degradation of demineralized dentin within a clinically relevant application time. In addition, the push-out bond strength of bonded restorations to dentin was also evaluated after dentin biomodification. The results after 6 months of water biodegradation were compared with those obtained immediately (after $24 \mathrm{~h}$ ). Additionally, the contact angle measurement of extract-treated dentin formed after adhesive application was analyzed. Extracts from mastruz (Dysphania ambrosioides), cat's claw (Uncaria tomentosa), guarana (Paullinia cupana), galla chinensis (Rhus chinensis galls), and tannic acid (obtained from Acacia decurrens) were used as dentin biomodifiers. The results were compared with a control, untreated group (negative control) and two positive controls: chlorhexidine $0.12 \%$ and tannic acid. The research hypotheses tested were (i) the bond strength of restorations filled with an adhesive restorative system will be negatively influenced by the previous application of plant extracts, irrespective of the evaluation time; (ii) the plant extracts would provide an increase in the elastic modulus of demineralized dentin; and (iii) the plant extracts will significantly affect the contact angle formed between the adhesive system and the dentin surface.

\section{Materials and Methods}

2.1. Preparation of Herbal Extracts. The different vegetable drugs $(0.750 \mathrm{~g})$ were subjected to extraction at $70^{\circ} \mathrm{C}$ for $30 \mathrm{~min}$ in $150 \mathrm{~mL}$ of distillated water (MTZ, CTC, and GUA). The decoction product was completed with distilled water up to $250 \mathrm{~mL}$. Afterward, about $80 \mathrm{~mL}$ of the solution was taken and filtered through filter paper, discarding the first $50 \mathrm{~mL}$. The filtrate obtained was called stock solution. The GCH solution was prepared by dissolving the commercially obtained extract in distilled water at a concentration of 4,000 ppm. TNA solution was also prepared in a concentration of $1 \%(\mathrm{w} / \mathrm{v})$. The experimental groups of the present study are described in Table 1.

2.2. Determination of Tannin Levels. To determine the tannin levels, a calibration curve regarding tannic acid was constructed. For that, an aqueous solution of tannic acid, $0.1 \mathrm{mg} /$ $\mathrm{mL}$, was prepared. Then, aliquots $(0.5,1.0,1.5,2.0,2.5$, and $3.0 \mathrm{~mL}$ ) were transferred to $100 \mathrm{~mL}$ volumetric balloons. The Folin-Ciocalteu phenol reagent $(5 \mathrm{~mL})$ was then added to $10 \mathrm{~mL}$ of the aqueous solution of sodium carbonate, and the volume was completed with distilled water. After $30 \mathrm{~min}$ (with standard temperature and lighting), each sample was read in the spectrophotometer at $760 \mathrm{~nm}$. After the dilutions, the final concentrations were $0.5,1.0,1.5,2.0,2.5$, and $3.0 \mu \mathrm{g} /$ $\mathrm{mL}$. The absorbance readings obtained at different concentrations of tannic acid were used to plot the calibration curve. Distilled water was used as a blank solution.

To determine the tannin levels in the plant extracts, the total phenol was initially quantified. For that, $1 \mathrm{~mL}$ of the extract was transferred to a $100 \mathrm{~mL}$ volumetric balloon containing $50 \mathrm{~mL}$ of distilled water. In the same way as employed for the construction of the curve, add $5 \mathrm{~mL}$ of the Folin-Ciocalteu phenol reagent, then add $10 \mathrm{~mL}$ of the aqueous solution of sodium carbonate, and complete the volume with distilled water. After $30 \mathrm{~min}$, the extracts were read in the spectrophotometer at $760 \mathrm{~nm}$. The residual phenol quantification was also evaluated. Casein $(1.0 \mathrm{~g})$ was transferred to a $50 \mathrm{~mL}$ Erlenmeyer flask, adding $6 \mathrm{~mL}$ of the extract and $12 \mathrm{~mL}$ of distilled water. After $3 \mathrm{~h}$ under agitation at room temperature, the extracts were filtered and read. For the total phenol quantification, this protocol was repeated. To estimate the tannin levels, the difference between the total phenol level and the noncomplex residual phenol level was calculated [13].

\subsection{UPLC/DAD-ESI/HRMS/MS Analytical Conditions.} Samples $(10 \mu \mathrm{L})$ from the different extracts were subjected to UPLC/DAD-ESI/HRMS/MS analysis. Thus, a Shimadzu chromatographic system, fitted with the Kinetex $2.6 \mathrm{~m} \mathrm{C}_{18}$ column and kept in the oven at $55^{\circ} \mathrm{C}$, was used. The analytical conditions were set as an exploratory gradient, starting with a low concentration of strong solvent, $15 \%$ (acetonitrile HPLC grade, with 1\% of formic acid), up to $95 \%$ 
TABLE 1: Vegetable drugs selected for the present study.

\begin{tabular}{|c|c|c|c|}
\hline Popular name & Scientific name & Source & Lot\# \\
\hline Mastruz (MTZ) & Dysphania ambrosioides & Leaves, bark, and stems & $\begin{array}{l}\text { 1737826333-8 } \\
\text { Panizza Laboratório } \\
\text { São Paulo, Brazil } \\
\text { 9816253436-3 }\end{array}$ \\
\hline Cat's claw (CTC) & Uncaria tomentosa & Leaves, bark, and stems & $\begin{array}{l}\text { Panizza Laboratório } \\
\text { São Paulo, Brazil } \\
\text { 1589538295-5 }\end{array}$ \\
\hline Guarana (GUA) & Paullinia cupana & Dry seeds & $\begin{array}{c}\text { Panizza Laboratório } \\
\text { São Paulo, Brazil } \\
\text { GCE99\%-20180628 }\end{array}$ \\
\hline Galla chinensis $(\mathrm{GCH})$ & Rhus chinensis & Dried extract & $\begin{array}{c}\text { Changsha Vigorous-tech Co. Ltd. } \\
\text { Hunan, China } \\
34783\end{array}$ \\
\hline Tannic acid (TNA) & Obtained from Acacia decurrens & Purified fraction & $\begin{array}{l}\text { Dinâmica laboratório } \\
\text { São Paulo, Brazil }\end{array}$ \\
\hline
\end{tabular}

in 12 minutes. The concentration remained constant for 4 minutes, returned to the initial conditions after $1 \mathrm{~min}$, and kept constant for $4 \mathrm{~min}$. The flow was set as $0.4 \mathrm{~mL} \cdot \mathrm{min}^{-1}$ and remained constant. The UPLC effluent was electrospray ionized and analyzed in positive and negative modes on the high-resolution mass spectrum, fitted with a QToF mass analyzer (Bruker Daltonics, Billerica, MA, USA). The drying gas temperature was defined as $200^{\circ} \mathrm{C}$ at a flow of $9 \mathrm{~L} \cdot \mathrm{min}^{-1}$, 2 bars for nebulizer pressure, and 4500 capillary voltage $(\mathrm{kV})$. To characterize the compounds, chromatographic band fragments with a mass charge ratio $(\mathrm{m} / \mathrm{z})$ between 50 and 1200 da were selected. Sodium formate was used as a calibrator. Data were treated using DataAnalysis 4.4 software (Bruker), and extracted ion chromatograms (EIC) were generated using Target Analysis 1.3 software and an in-house Excel list of target candidates, with the compound names and molecular formulas, according to the chemical composition literature for each plant species and/or genus [14-19].

2.4. Compressive Bond Strength Test. Seventy bovine incisors were selected, cleaned, and stored in a $0.5 \%$ chloramine $\mathrm{T}$ solution at $4^{\circ} \mathrm{C}$. The specimens were ground flat to obtain $2 \mathrm{~mm}$ thick slices as previously described [20]. The specimens were randomly distributed into 6 groups $(n=10)$ according to the dentin biomodification treatment: (1) control (CTL, distilled water), (2) chlorhexidine $0.12 \%$ (CHX), (3) mastruz (MTZ), (4) cat's claw (CTC), (5) guarana (GUA), (6), galla chinensis $(\mathrm{GCH})$, and (7) tannic acid (TNA).

Conical preparations were performed in the specimens with margins in dentin (top diameter $4.0 \mathrm{~mm}$, bottom diameter $3.0 \mathrm{~mm}, 2.0 \mathrm{~mm}$ thick). The dentin slices were acidetched for $15 \mathrm{~s}$ with $35 \%$ phosphoric acid (Scotchbond Etchant; 3M ESPE, St. Paul, MN, USA) and rinsed for $20 \mathrm{s.}$ The plant extracts were applied for $60 \mathrm{~s}$ using a microbrush. The excess solution was dried with absorbent paper. Then, the adhesive system (lot \#N808310, Adper Single Bond 2; 3M ESPE, St. Paul, MN, USA) was applied and cured for $10 \mathrm{~s}$. The specimens were positioned over a polyester strip. The composite (lot \#18177008007, Filtek Z350XT; 3M ESPE, St. Paul, MN, USA) was inserted into the preparations. The preparations were filled with the composite and then covered with a Mylar strip and then compressed with a glass slide. The composite was then photoactivated for $20 \mathrm{~s}$ $\left(1200 \mathrm{~mW} / \mathrm{cm}^{2}\right.$, Bluephase; Ivoclar Vivadent, Schaan, Liechtenstein). The specimens were then stored at $37^{\circ} \mathrm{C}$ in physiological saline solution for $24 \mathrm{~h}$. Restorations were finished and polished using abrasive discs (Sof-Lex; 3M ESPE, St. Paul, MN, USA).

The compressive push-out bond strength of bonded restorations was evaluated according to the following factors: (1) dentin pretreatment at two levels: control, untreated dentin, and dentin biomodification with plant extracts and (2) storage time at two levels: immediate $(24 \mathrm{~h})$ and after 6 months. Seven experimental groups were categorized $(n=10)$. The push-out bond strength test was conducted as previously described [20], at a crosshead speed of $0.5 \mathrm{~mm} /$ min. The compressive force of the probe was applied at the bottom surface of the restoration. The means were expressed in $\mathrm{MPa}$ [20]. After the test, a fractographic analysis was also performed at $5 \times$ magnification using a dissecting microscope (Stereozoom; Bausch \& Lomb, Rochester, NY, USA) according to a previously described classification [20].

2.5. Modulus of Elasticity of Demineralized Dentin after Dentin Biomodification. Specimens were obtained as previously described $[21,22]$. Briefly, sections of bovine incisor teeth were obtained $(0.5 \times 1.7 \times 7.0 \mathrm{~mm})$ using a watercooled rotating diamond wheel (IsoMet; Buehler Ltd., Evanston, IL, USA). The specimens were then immersed in $10 \%$ phosphoric acid solution (LabChem, Pittsburgh, PA, USA) for $5 \mathrm{~h}$ and then thoroughly rinsed with distilled water for $10 \mathrm{~min}$. The specimens were then immersed in distilled water for baseline measurements. Before the treatment, the initial dry weight and initial flexural modulus (three-point bending test) were assessed as previously described [21]. The demineralized specimens $(n=15)$ were treated with plant extracts for $60 \mathrm{~s}$ [23]. Then, the specimens were vigorously rinsed with distilled water for $30 \mathrm{~s}$. The flexural modulus was 
reassessed immediately after immersion, and the treated beams were individually stored in $1 \mathrm{~mL}$ of artificial saliva (pH 7.4), containing HEPES $5 \mu \mathrm{M}, \mathrm{CaCl}_{2} 2.5 \mathrm{mM}, \mathrm{ZnCl}_{2}$ $0.05 \mathrm{mM}$, and $\mathrm{NaCl} 120 \mathrm{mM}$, at $37^{\circ} \mathrm{C}$ for four weeks to undertake collagen degradation [24].

The specimens were tested on three-point bending setup in a universal testing machine (Instron 4484; Instron Inc., Canton, USA), with a $5 \mathrm{~N}$ load cell at $0.5 \mathrm{~mm} / \mathrm{min}$ crosshead speed. Load-displacement curves were converted to stressstrain curves. The width and thickness of the specimens were measured, and the elastic modulus was calculated at 3\% strain as previously described [21]. Data were expressed in $\mathrm{MPa}$, and the percentage variation in elastic modulus was calculated as the ratio of the final value (after treatment with plant extracts) to the initial values (baseline).

2.6. Mass Weight Change of Demineralized Dentin after Dentin Biomodification. Demineralized dentin beams were weighted before $\left(M_{1}\right)$ and after $\left(M_{2}\right)$ dentin biomodification with an analytical balance $(0.00001 \mathrm{mg}$ precision; Ohaus Analytical Plus, Florham Park, NJ, USA). An additional mass weight assessment was performed after 4 weeks of degradation in artificial saliva $\left(M_{3}\right)$. In each period, the specimens were initially dried in a vacuum desiccator containing silica gel beads for $72 \mathrm{~h}$ at room temperature. Mass weight variation $\left(W_{m c} \%\right)$ was determined as the percentage of gain or loss in mass for each specimen, as previously described [21], based on the following formula for biomodification:

$$
W_{m c}(\%)=\left[\frac{M_{2} \times 100}{M_{1}}\right]-100,
$$

where $M_{1}$ is the demineralized dentin beam mass weight before dentin biomodification and $M_{2}$ is the mass weight of the biomodified dentin matrix. Moreover, to assess the biodegradation percentage mass weight variation, the following formula was used:

$$
W_{d e}(\%)=\frac{\left[\left(M_{3}-M_{1}\right) \times 100\right]}{M_{1}},
$$

where $M_{1}$ is the demineralized dentin beam mass weight before dentin biomodification and $M_{3}$ is the mass weight of dentin matrix after four weeks of artificial saliva immersion.

2.7. Surface Wettability Assessment. Two-millimeter-thick dentin slabs were obtained from bovine incisor teeth by grinding the enamel surface to a 600-grit finish with $\mathrm{SiC}$ paper until flat dentin areas were obtained (around $5 \mathrm{~mm}$ of width $\times 6 \mathrm{~mm}$ of length). The smear layer was kept intact for the contact angle measurements. The specimens were immersed in distilled water until analysis. As previously described, dentin slices were acid-etched for $15 \mathrm{~s}$ with $35 \%$ phosphoric acid and water-rinsed for $20 \mathrm{~s}$. The plant extracts were applied for $60 \mathrm{~s}$ using a microbrush. The excess solution was dried with absorbent paper. Then, one drop $(5 \mu \mathrm{L})$ of the adhesive system was deposited using a micropipette (Eppendorf, Hamburg, Germany) onto each dentin slice after dentin biomodification with the plant extracts. Digital images of the specimens were obtained by taking horizontal photographic shots at right angle to the long axis of the dentin with a high-resolution (5.0 megapixel) digital still camera, Nikon 90 (Nikon Inc, Japan), attached to a lens (Nikon Medical-Nikkor, $120 \mathrm{~mm} \mathrm{f} / 4 \mathrm{IF}$ ), positioned $30 \mathrm{~mm}$ from the specimen. High-quality images in JPEG (Joint Photographic Experts Group) format at maximum quality and without changes were obtained with an $8 \times$ magnification. An angular dimension measuring tool was used to measure the contact angle $(\theta)$ formed by the contact surface of the dentin specimens and the tangent of the adhesive drop (https://www.ginifab.com/feeds/angle_measurement/). Right and left angle measurements $(n=3)$ were obtained, and then an average was calculated for each specimen. A control, untreated group was also evaluated.

2.8. Statistical Analysis. Means and standard deviations of the compressive bond strength test were calculated and statistically analyzed with two-way ANOVA and Tukey's post hoc test, with a preset alpha of 5\%. Data of elastic modulus, mass weight variation, and contact angle analysis were statistically analyzed with ANOVA/Tukey's test (5\% of significance).

\section{Results}

In Table 2 are displayed the tannin levels for different studied species, illustrating the individual variations. In general, tannin levels can be ranked as follows: $\mathrm{GCH}>\mathrm{TNA}>\mathrm{GUA}>\mathrm{CTC}>\mathrm{MTZ}$ (Table 2).

Chromatograms of each plant extract are presented in Figure 1, while the compounds identified within the respective retention times $\left(R_{t}\right)$, molecular ion masses $(\mathrm{m} / z)$, and relative peak area (RPA) are summarized in Table 3.

Gallic acid $\left(R_{t}=0.8 \mathrm{~min} ; \mathrm{m} / z \quad 169.0139-[\mathrm{M}-\mathrm{H}]^{-}\right.$; $\mathrm{RPA}=36.6 \%)$ and methoxybenzoic acid derivative $\left(R_{t}=3.9 \mathrm{~min} ; m / z 151.0570-[\mathrm{M}-\mathrm{H}]^{-} ; \mathrm{RPA}=26.8 \%\right)$ were identified at GCH and caffeine $\left(R_{t}=1.3 \mathrm{~min} ; \mathrm{m} / z 195.0881-\right.$ $\left.[\mathrm{M}+\mathrm{H}]^{+} ; \mathrm{RPA}=84.3 \%\right)$ at GUA. At MTZ were observed ions of organic acids - malic and citric acids $\left(R_{t}=0.7 \mathrm{~min}\right.$; $m / z 133.0145$ and $\left.191.0193-[\mathrm{M}-\mathrm{H}]^{-} ; \mathrm{RPA}=77.7 \%\right)$, and those organic acids were also observed at CTC, within quinic acid $\left(R_{t}=0.7 \mathrm{~min} ; \mathrm{m} / z 191.0562-[\mathrm{M}-\mathrm{H}]^{-} ; \mathrm{RPA}=10.5 \%\right)$; however, the major compound identified at CTC was chlorogenic acid $\left(R_{t}=0.9 \mathrm{~min} ; \mathrm{m} / z \quad 353.0879-[\mathrm{M}-\mathrm{H}]^{-}\right.$; $\mathrm{RPA}=58.0 \%)$. Tannic acid presents a molecular weight higher than the upper limit of MS detector specification $(m / z>1200)$. Although it was not possible to observe the molecular ion, some characteristic fragmentation ions were observed for TNA at negative mode $-m / z$ 169.0147, 369.0290, and 673.0898 [25].

The results of the push-out bond strength test are described in Table 4 . The highest push-out bond strength mean was observed for CTC after 6 months $(42.8 \mathrm{MPa})$, and the lowest when treated with CHX was evaluated after $24 \mathrm{~h}$ (34.2 MPa). No significant differences were observed among the experimental groups, regardless of the dentin treatment 
TABLe 2: Extracts analyzed with respect to tannin level (\% w/w).

\begin{tabular}{lc}
\hline Extract & Tannin $(\%)$ \\
\hline MTZ & $\mathbf{0 . 0}(0.0)$ \\
CTC & $\mathbf{9 . 5}(0.9)$ \\
GUA & $\mathbf{1 0 . 8}(0.5)$ \\
GCH & $\mathbf{3 3 . 7}(0.6)$ \\
TNA & $\mathbf{2 6 . 2}(2.6)$ \\
\hline
\end{tabular}

Values within the parenthesis are standard deviation.

and the evaluation time $(p>0.05)$. Type 1 failure mode (adhesive mode) was the most frequent, irrespective of the factors evaluated (Figure 2).

Table 5 shows the mean dentin elastic modulus and the standard deviation. The statistical analysis demonstrated significant differences between the TNA-treated group and the experimental groups $(p<0.05)$. TNA induced an increase of $70.8 \%$ of the dentin elastic modulus. Except for dentin specimens treated with TNA, the modulus significantly decreased after dentin treatment with the extract plants (from $-2.2 \%$ for CTC to $-49.4 \%$ for CHX). After biodegradation in artificial saliva, a significant increase in the elastic modulus was observed for the groups treated with GCH and TNA compared with the control groups (CTL and CHX) and to the other dentin treatments $(p<0.05)$. There was a significant increase of the mass for the experimental groups treated with CHX, MTZ, GUA, and GCH (Table 6). After biodegradation in artificial saliva, all experimental groups exhibited dentine mass reduction, which was significantly higher for the CTL group and for the groups treated with MTZ, CTC, and TNA (Table 6). Representative pictures of the specimens after biodegradation are presented in Figure 3. Darker color was observed in specimens treated with GCH and TNA, whereas those treated with the other extracts demonstrated reduced, but with clear signs of pigmentation.

Contact angles of the adhesive with the extract-treated dentin are demonstrated in Table 7. The highest contact angle was observed in the CTL group (38.0 degrees), and the lowest (20.7 degrees) was observed when TNA was applied previously to the adhesive system. The application of CTC, $\mathrm{GCH}$, and TNA significantly decreased the contact angle compared with the control group $(p<0.05)$.

\section{Discussion}

The use of biomodifying agents was proposed to provide stabilization and strengthening of the collagen matrix by improving the mechanical properties of the dentin [6]. In this manner, these agents are regarded to increase the bond strength of bonded restorations to dentin, to reduce the biodegradation rate of demineralized collagen, and consequently, to increase the longevity of adhesive restorations $[4,26]$. Cross-linking agents are claimed to react in different ways with collagen, creating different kinds and extents of cross-links, mainly via bonding with hydrogen ions (with natural agents) and covalent bonds (with synthetic agents) $[6,27]$. Plant extracts have also been used to induce dentin biomodification mainly due to the presence of tannins, especially proanthocyanidins [28]. Tannins are polyphenolic compounds that are associated with numerous secondary activities in plants [29]. Tannins can be used as a potential cross-linker for the development of biomaterials for clinical applications [30]. Among several actions, tannins were found to have an affinity for type I collagen [8], the most abundantly found in dentin, which is exposed after acid conditioning. Tannins are regarded to bind to collagen, inducing significant changes in the conformation of the triple helix of type I collagen, thus increasing its structural, thermal, and enzymatic stability [8].

The plant drugs selected in the present study presented complex mixtures of compounds (Figure 1). Phenols and polyphenols such as gallic acid, methoxybenzoic derivative, and chlorogenic acid, found in plant materials, were detected in the extracts of GCH and CTC. While caffeine was identified in GUA extract, other organic acids such as malic, citric, and quinic acids were identified in MTZ and CTC. TNA, a commercial form of condensed tannin, is a naturally occurring collagen cross-linking agent consisting of a complex mixture of polygalloylglucose esters with weak acidity [31, 32]. Although most studies in which the biomodifying action of dentin with plant drugs was evaluated focused on the presence of tannins, other bioactive agents with lower molecular weight can also act as biomodifiers, inducing collagen cross-linking [26].

The dentin biomodification effect of bioactive agents found in the plant extracts had no influence on the bond strength of bonded restorations to dentin, regardless of the evaluation period $(p>0.05)$. In this manner, the first hypothesis, which anticipated that the bond strength to dentin would be negatively influenced by the previous application of extract plants, was not accepted. Conversely, except when treated with TNA, the mechanical properties of exposed dentinal collagen were negatively influenced by all dentin treatments with the plant extracts $(p>0.05)$. Thus, the second hypothesis in which it was speculated that the extract plants would provide an increase in the elastic modulus of demineralized dentin was not accepted.

Tannin-rich extracts as cross-linkers prior to the application of adhesive restorative materials demonstrated superior results when compared with chlorhexidine [33-36]. Conversely, concerns have been expressed over their actual benefits, especially when cross-linkers are applied in clinically relevant application times [27]. It has been questioned that the ability of a cross-linking agent to react with the dentin matrix to avoid the degradation of collagen at the adhesive interfacial area depends on its chemical and structural characteristics [27]. In the present study, only TNA proved to be equally effective in chemically modifying collagen, which explains the reason to use it as a positive control. A previous study demonstrated that TNA is able to reduce the enzymatic degradation to improve the mechanical properties of dentin, thus increasing the bond strength of resin-based restorations to dentin [32].

The fact that the plant extracts reduced the modulus of elasticity after treatment, in a certain way, demonstrated that these extracts were not able to induce collagen cross-linking. Although the compounds found in tannin-rich plant extracts seem to present multiple phenolic functionalities, 


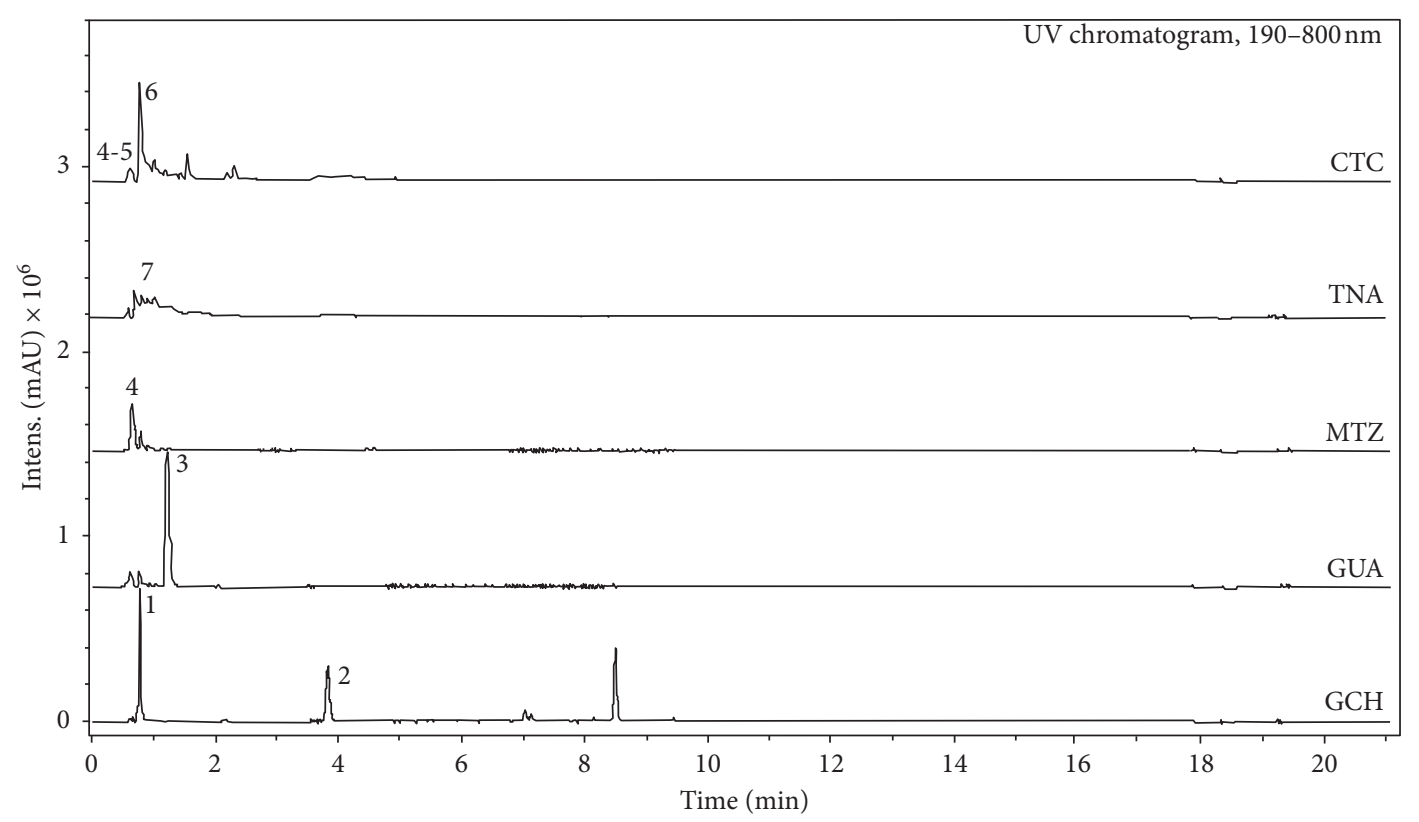

FIGURE 1: Phytochemical profile (fingerprint) of the plant extracts through hyphenated technique (UPLC/DAD-ESI/HRMS/MS).

TABLE 3: Major compounds identified in plant extracts with UPLC/DAD-ESI/HRMS/MS.

\begin{tabular}{|c|c|c|c|c|c|}
\hline$\#^{*}$ & Compound name & $R_{t}(\min )$ & $m / z(\mathrm{Da})$ & RPA (\%) & Extract \\
\hline 1 & Gallic acid & 0.8 & 169.0139 & 36.6 & $\mathrm{GCH}$ \\
\hline 2 & Methoxybenzoic acid derivative & 3.9 & 151.0570 & 26.8 & $\mathrm{GCH}$ \\
\hline 3 & Caffeine & 1.3 & 195.0881 & 84.3 & GUA \\
\hline 4 & $\begin{array}{l}\text { Malic acid } \\
\text { Citric acid }\end{array}$ & $\begin{array}{l}0.7 \\
0.7\end{array}$ & $\begin{array}{l}133.0145 \\
191.0193\end{array}$ & 77.7 & MTZ; CTC \\
\hline 5 & Quinic acid & 0.7 & 191.0562 & 10.5 & CTC \\
\hline 6 & Chlorogenic acid & 0.9 & 353.0879 & 58.0 & CTC \\
\hline 7 & Tannic acid & - & $>1200$ & - & TNA \\
\hline
\end{tabular}

${ }^{*}$ Number of compounds identified in Figure 1. Rt: retention time; RPA: relative peak area.

TABle 4: Push-out strength means as a function of the evaluation time.

\begin{tabular}{lccccccc}
\hline & CTL & CHX & MTZ & CTC & GUA & GCH \\
\hline 24 hours & $39.1^{\text {a.A }}(15.7)$ & $34.2^{\text {a.A }}(11.8)$ & $41.1^{\text {a.A }}(20.8)$ & $42.0^{\text {a.A }}(18.5)$ & $35.3^{\text {a.A }}(15.3)$ & $39.9^{\text {a.A }}(13.7)$ & $42.6^{\text {a.A }}(9.4)$ \\
6 months & $36.2^{\text {a.A }}(14.0)$ & $34.8^{\text {a.A }}(18.1)$ & $41.7^{\text {a.A }}(12.5)$ & $42.8^{\text {a.A }}(10.5)$ & $39.4^{\text {a.A }}(10.9)$ & $40.2^{\text {a.A }}(6.3)$ & $40.0^{\text {a.A }}(8.2)$ \\
\hline
\end{tabular}

Mean values followed by different capital letters in row and small letters in column indicate significant differences $(p<0.05)$. Values within the parenthesis are standard deviation.

oligomeric structures that have high biomodifying potential may present higher molecular weight [37]. This helps explain their slower ability to diffuse in the collagen matrix, explaining the lesser influence on the elastic modulus and resistance to degradation when applied for 1 minute [23]. It has been also claimed that changes in the elastic modulus induced by the plant extracts should be associated with its ability to nucleate calcium and phosphate to form amorphous calcium phosphate crystals to remineralize the exposed uninfiltrated collagen [3]. Thus, it can be speculated that the true biomodifying action of the extracts after treatment of the dentin fragments should be evaluated after immersion in a saturated solution of calcium and phosphate $(\mathrm{Ca} / \mathrm{P}$ ratio $=1.67)[3]$. This fact can be corroborated by the "late" biomodifying action of galla chinensis, which induced an increased elastic modulus after immersion in calciumcontaining artificial saliva.

The interactions and complexation of the compounds of the extracts with dentin collagen also seem to explain the variations in mass (gain or loss) observed in the present study (Table 4). In addition, the resistance to biodegradation is related not only to the increase in the modulus of elasticity induced by the formation of collagen cross-linking but also to the hydrophilicity of the biomodifying agents [38]. This also explains the stability of the bond strength in the experimental groups after 6 months. In a previous study, it was highlighted that the ability of the plant extracts to dehydrate collagen fibrils by means of the cross-linking effect would 


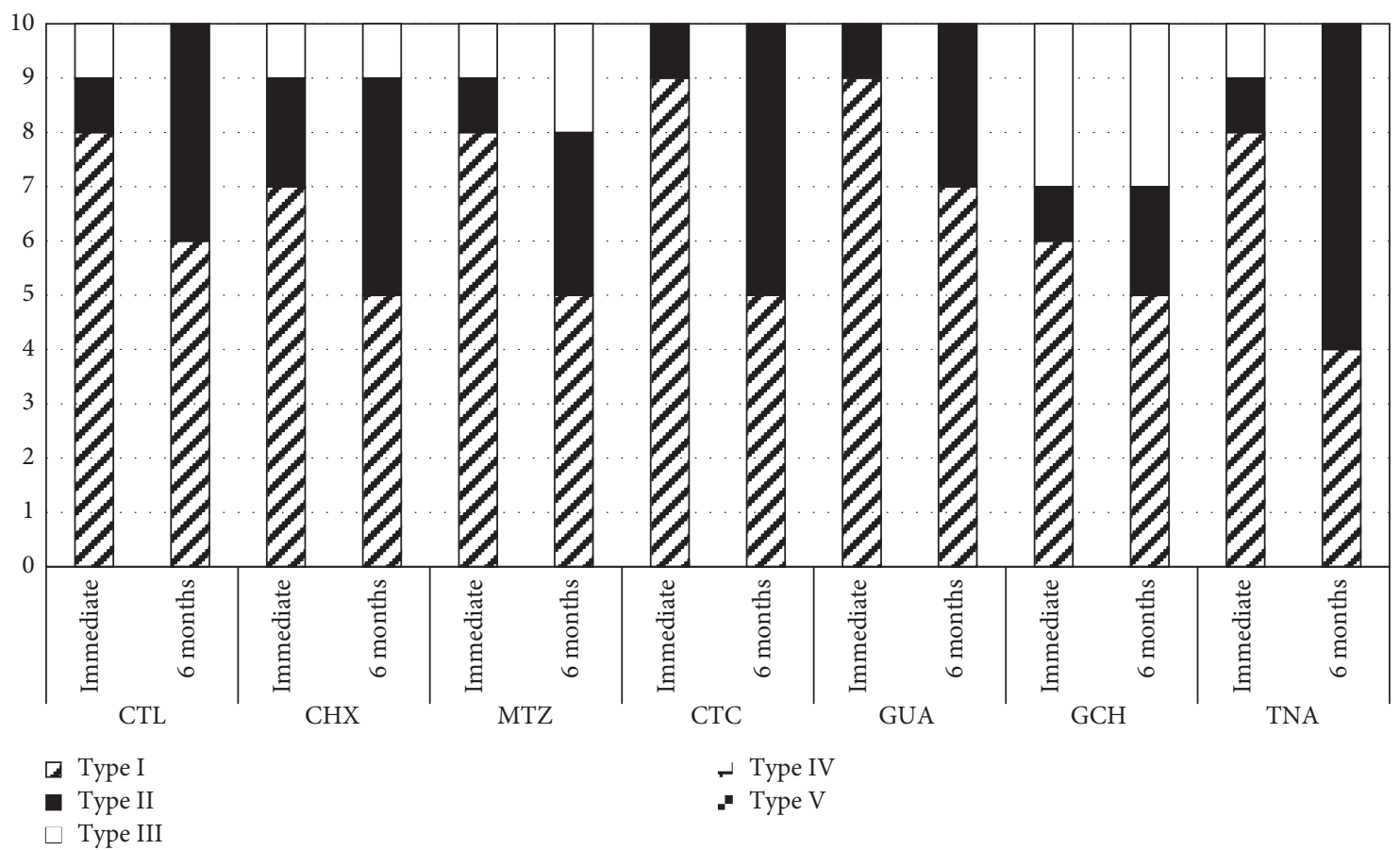

FIGURE 2: Incidence of failure modes (\%) according to the experimental groups.

TABle 5: Means (standard deviation) of modulus of elasticity (in MPa) of demineralized dentin as a function of the treatment.

\begin{tabular}{lccc}
\hline $\begin{array}{l}\text { Experimental } \\
\text { groups }\end{array}$ & $\begin{array}{c}\text { Initial modulus } \\
(\mathrm{MPa})\end{array}$ & $\begin{array}{c}\text { Elastic modulus variation after dentin } \\
\text { biomodification }(\%)\end{array}$ & $\begin{array}{c}\text { Elastic modulus variation after biodegradation in } \\
\text { artificial saliva }(\%)\end{array}$ \\
\hline CTL & $2.04^{\mathrm{A}}(0.95)$ & $-27.5^{\mathrm{B}}(32.2)$ & $-40.7^{\mathrm{B}}(25.5)$ \\
CHX & $2.81^{\mathrm{A}}(1.12)$ & $-49.4^{\mathrm{B}}(29.4)$ & $-37.1^{\mathrm{B}}(47.5)$ \\
MTZ & $3.15^{\mathrm{A}}(2.22)$ & $-34.7^{\mathrm{B}}(47.8)$ & $-50.0^{\mathrm{B}}(44.3)$ \\
CTC & $2.14^{\mathrm{A}}(0.96)$ & $-2.2^{\mathrm{B}}(51.0)$ & $-28.1^{\mathrm{B}}(31.3)$ \\
GUA & $2.39^{\mathrm{A}}(1.52)$ & $-21.4^{\mathrm{B}}(29.7)$ & $-28.7^{\mathrm{B}}(32.3)$ \\
GCH & $2.45^{\mathrm{A}}(1.36)$ & $-3.9^{\mathrm{B}}(41.1)$ & $6.8^{\mathrm{A}}(32.3)$ \\
TNA & $2.41^{\mathrm{A}}(1.38)$ & $70.8^{\mathrm{A}}(87.9)$ & $27.6^{\mathrm{A}}(72.1)$ \\
\hline
\end{tabular}

Different capital letters (A and B) for comparisons between lines indicate significant difference $(p<0.05) . N=15$.

TABle 6: Mean (SD) of mass variation (\%) of dentin specimens as a function of the treatment.

\begin{tabular}{lcc}
\hline Experimental groups & Mass variation (\%) after dentin biomodification & Mass variation (\%) 4 weeks of degradation \\
\hline CTL & $-4.8(6.3)^{\mathrm{B}}$ & $-16.8(6.9)^{\mathrm{B}}$ \\
CHX & $+7.2(3.6)^{\mathrm{A}}$ & $-7.0(6.0)^{\mathrm{A}}$ \\
MTZ & $+0.7(13.4)^{\mathrm{B}}$ & $-10.0(14.8)^{\mathrm{B}}$ \\
CTC & $-0.6(3.9)^{\mathrm{B}}$ & $-12.2(4.9)^{\mathrm{B}}$ \\
GUA & $+3.3(3.9)^{\mathrm{A}}$ & $-7.8(5.2)^{\mathrm{A}}$ \\
GCH & $+1.0(4.6)^{\mathrm{B}}$ & $-4.9(6.1)^{\mathrm{A}}$ \\
TNA & $-2.5(2.8)^{\mathrm{B}}$ & $-8.5(13.6)^{\mathrm{B}}$ \\
\hline
\end{tabular}

Different letters within each column indicate statistically significant difference $(p<0.05) . N=15$.

render a more promising collagen substrate for hybridization and eventually a more stable hybrid layer [39]. On the other hand, it has been pointed out that hydrophobic bioactive agents can also induce less degradation of the collagen matrix when compared with hydrophilic agents [23]. Biomodifying agents were also regarded to induce the formation of cross-links in the dentin collagen during the formation of chemical bonds between the cross-linking agents and the collagen. It has been advocated that these agents would remove proteoglycans from the dentin matrix, replace the water bound to the collagen fibrils, and reduce its hydrophilicity [38]. In this manner, these agents would favor a 


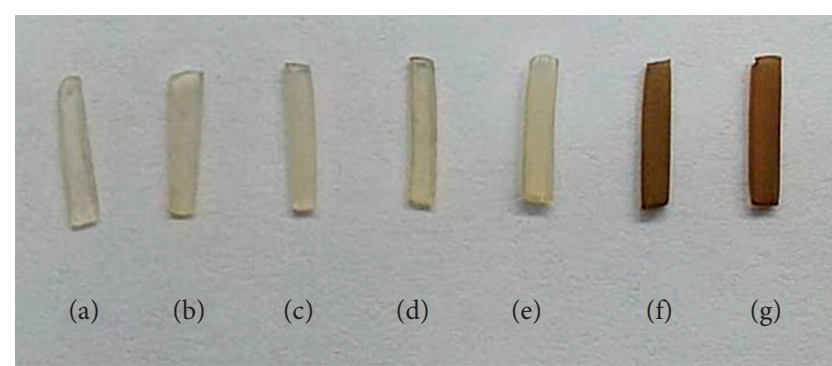

FIgURE 3: Color changes in demineralized dentin specimens after biodegradation in artificial saliva for four weeks. (a) CTL; (b) CHX; (c) MTZ; (d) CTC; (e) GUA; (f) GCH; and (g) TNA.

TABLE 7: Contact angle formed by the adhesive system on dentin treated with different plant drug extracts.

\begin{tabular}{lc}
\hline Experimental groups & Contact angle* \\
\hline CTL & $38.0(2.0)^{\mathrm{A}}$ \\
CHX & $31.3(0.3)^{\mathrm{A}}$ \\
MTZ & $36.3(0.3)^{\mathrm{A}}$ \\
CTC & $25.8(0.2)^{\mathrm{B}}$ \\
GUA & $29.2(1.2)^{\mathrm{A}}$ \\
GCH & $24.7(0.7)^{\mathrm{B}}$ \\
TNA & $20.7(0.3)^{\mathrm{B}}$ \\
\hline
\end{tabular}

Different letters (A and B) within the columns indicate significant difference $(p<0.05) .{ }^{*}$ In degrees.

greater diffusion of monomers of the adhesive system through the collagen network, allowing it fully expand [40], reducing the rates of biodegradation $[4,6]$.

In a certain way, the dentin wettability after treatment with the plant extracts, which would improve the permeation of the adhesive system and the longevity of the adhesive interfaces, needs to be further confirmed to certify the action of these biomodifying agents. At a molecular level, the intermolecular spaces between the collagen fibrils are preserved by the water surrounding the triple helices of the collagen molecules [3]. Consequently, hydrogen bridges are formed preventing direct contact between chains, thus avoiding collagen collapse [41]. For the adhesive to have an effective bond to the dentin, it is necessary to have a network structure of noncollapsed collagen fibrils, with interfibrillar spaces filled with water, allowing the proper infiltration of resin monomers in the demineralized intertubular dentin, to then form the hybrid layer [42]. The fact that the plant drugs of the present study are water-soluble seems to have favored the dentin wettability prior to the adhesive system. The contact angle of the adhesive with dentin was significantly reduced when treated with the plant extracts CTC, GCH, and TNA in comparison with the control groups (CTL and CHX). In this manner, the third hypothesis, which speculated that the extract plants would significantly affect the contact angle formed between the adhesive system and the dentin surface, was partially accepted.

In addition, considering the clinical application of the plant extracts in dentin, it was observed that the greatest changes in the color of the dentin fragments correlated with the highest concentration of tannins in them. This in a way makes its clinical use unfeasible. In spite of the favorable results of GCH in remineralizing dental tissues in several studies $[43,44]$, dental products with this appeal are certainly not available so far because of the dentin color change. It has been widely known that green tea, a popular drink consumed daily by millions of people worldwide, is known to cause blackening of the teeth because it is rich in epigallocatechin gallate (65\% of its composition) [45]. This was also observed in another previous study in which a tanninrich bioactive agent stained the dentin specimens intensely in the dark brown shade [23]. The present study demonstrated that the plant drug extracts tested had different effects on dentin and more specifically on dentin collagen. Different from that of other studies, a questionable effect of the plant extracts with variable concentration of tannins as dentin biomodifiers was demonstrated. Although the extracts of plant drugs had no impact on the bond strength, the dentin modulus was significantly reduced after the dentin treatment with the extract plants. In addition, the use of tannin-rich extracts prior to adhesive application was proven to be clinically unfeasible due to dentin staining. The highest tanning content in the extracts induced darker staining, which is favored by the tubular structure of the dentin, diffused by capillarity through other regions of the dentinal structure.

Considering its limitations, the present study was conducted in vitro with the extracts. In addition, it was not clear how long is the duration of the contact of the tannins and other compounds of plant extracts with the dentin collagen fibrils. This could influence the binding mechanism of tannins to collagen fibrils and their potential stabilization mechanism [3]. In spite of these limitations, these results provided important new information regarding the application of these plant extracts previously to the adhesive bonding. Furthermore, the results of a previous study corroborate with our results, which proved that tannic acid (TNA) affects the mechanical properties of demineralized dentin by increasing its stiffness and provides collagen stabilization [32]. Future studies are necessary to provide more information, including the evaluation of the effect of these tannin-rich plant extracts on the nanostructure of collagen fibrils and in vivo studies. In spite of the clinically negative impact of dentin staining, some of the plant extracts were able to change the dentin wettability, as demonstrated by the reduction in contact angle. This seems to favor a greater diffusion and permeation of the resinous monomers of the adhesive throughout the collagen fibrils.

\section{Conclusions}

Within the limitations of this study, it can be concluded that although the bioactive plant extracts had no impact on the bond strength to dentin regardless of the evaluation time, it was observed a negative influence on the apparent modulus of elasticity of the demineralized dentin. Some of the extracts changed the contact angle of the adhesive with the dentin, possibly improving the adhesive permeation throughout the demineralized dentin. Conversely, the higher the tannin content, the more the extracts stained the dentin, which is 
impeditive for its clinical application. Further studies are needed to understand the mechanisms involved in the intimacy of the tooth-restoration interface when using the evaluated plant drug extract.

\section{Data Availability}

The data used to support the findings of this study are available from the corresponding author upon request.

\section{Disclosure}

This study was developed as a partial fulfillment of the requirements of Dr. Polassi's Master degree.

\section{Conflicts of Interest}

The authors declare that there are no conflicts of interest regarding the publication of this study.

\section{Acknowledgments}

This study was partially supported by grants from CAPES and CNPq (grant \#400847/2018-3), Brazil. The authors are grateful to Universidade Anhanguera de São Paulo (UNIAN-SP) for technical support.

\section{References}

[1] B. Oliveira-Reis, A. T. Maluly-Proni, T. C. Fagundes et al., "Influence of protease inhibitors on the degradation of sound, sclerotic and caries-affected demineralized dentin," Journal of the Mechanical Behavior of Biomedical Materials, vol. 97, pp. 1-6, 2019.

[2] A. Frassetto, L. Breschi, G. Turco et al., "Mechanisms of degradation of the hybrid layer in adhesive dentistry and therapeutic agents to improve bond durability-a literature review," Dental Materials, vol. 32, no. 2, pp. e41-e53, 2016.

[3] R. G. Carvalho, M. M. P. Alvarez, T. de Sá Oliveira et al., "The interaction of sodium trimetaphosphate with collagen I induces conformational change and mineralization that prevents collagenase proteolytic attack," Dental Materials, vol. 36, no. 6, pp. e184-e193, 2020.

[4] L. Tjäderhane, F. D. Nascimento, L. Breschi et al., "Optimizing dentin bond durability: control of collagen degradation by matrix metalloproteinases and cysteine cathepsins," Dental Materials, vol. 29, no. 1, pp. 116-135, 2013.

[5] L. Breschi, A. Mazzoni, A. Ruggeri, M. Cadenaro, R. Di Lenarda, and E. De Stefano Dorigo, "Dental adhesion review: aging and stability of the bonded interface," Dental Materials, vol. 24, no. 1, pp. 90-101, 2008.

[6] A. K. Bedran-Russo, G. F. Pauli, S.-N. Chen et al., "Dentin biomodification: strategies, renewable resources and clinical applications," Dental Materials, vol. 30, no. 1, pp. 62-76, 2014.

[7] M.-P. Morin and D. Grenier, "Regulation of matrix metalloproteinase secretion by green tea catechins in a three-dimensional co-culture model of macrophages and gingival fibroblasts," Archives of Oral Biology, vol. 75, pp. 89-99, 2017.

[8] P. Velmurugan, E. R. A. Singam, R. R. Jonnalagadda, and V. Subramanian, "Investigation on interaction of tannic acid with type I collagen and its effect on thermal, enzymatic, and conformational stability for tissue engineering applications," Biopolymers, vol. 101, no. 5, pp. 471-483, 2014.
[9] B. Han, J. Jaurequi, B. W. Tang, and M. E. Nimni, "Proanthocyanidin: a natural crosslinking reagent for stabilizing collagen matrices," Journal of Biomedical Materials Research, vol. 65A, no. 1, pp. 118-124, 2003.

[10] A. Mazzoni, L. Tjäderhane, V. Checchi et al., "Role of dentin MMPs in caries progression and bond stability," Journal of Dental Research, vol. 94, no. 2, pp. 241-251, 2015.

[11] I. L. Tersariol, S. Geraldeli, C. L. Minciotti et al., "Cysteine cathepsins in human dentin-pulp complex," Journal of Endodontics, vol. 36, no. 3, pp. 475-481, 2010.

[12] M. T. Kato, A. L. Leite, A. R. Hannas et al., "Impact of protease inhibitors on dentin matrix degradation by collagenase," Journal of Dental Research, vol. 91, no. 12, pp. 1119-1123, 2012.

[13] E. L. C. Amorim, J. E. Nascimento, J. M. Monteiro et al., “A simple and accurate procedure for the determination of tannin and flavonoid levels and some applications in ethnobotany and ethnopharmacology," Functional Ecosystems and Communities, vol. 2, pp. 88-94, 2008.

[14] F. Tian, B. Li, B. Ji et al., "Antioxidant and antimicrobial activities of consecutive extracts from Galla chinensis: the polarity affects the bioactivities," Food Chemistry, vol. 113, no. 1, pp. 173-179, 2009.

[15] F. Tian, B. Li, B. Ji, G. Zhang, and Y. Luo, "Identification and structure-activity relationship of gallotannins separated from Galla chinensis," LWT-Food Science and Technology, vol. 42, no. 7, pp. 1289-1295, 2009.

[16] L. Barros, E. Pereira, R. C. Calhelha et al., "Bioactivity and chemical characterization in hydrophilic and lipophilic compounds of Chenopodium ambrosioides L." Journal of Functional Foods, vol. 5, no. 4, pp. 1732-1740, 2013.

[17] M. E. Heitzman, C. C. Neto, E. Winiarz et al., "Ethnobotany, phytochemistry and pharmacology of (Rubiaceae)," Phytochemistry, vol. 66, no. 1, pp. 5-29, 2005.

[18] B. C. Azevedo, L. J. F. Morel, F. Carmona et al., "Aqueous extracts from Uncaria tomentosa (Willd. ex Schult.) DC. reduce bronchial hyperresponsiveness and inflammation in a murine model of asthma," Journal of Ethnopharmacology, vol. 218, pp. 76-89, 2018.

[19] G. S. da Silva, K. M. Canuto, P. R. V. Ribeiro et al., "Chemical profiling of guarana seeds (Paullinia cupana) from different geographical origins using UPLC-QTOF-MS combined with chemometrics," Food Research International, vol. 102, pp. 700-709, 2017.

[20] P. J. d. Santos, M. S. Silva, R. C. B. Alonso, and P. H. P. D’Alpino, "Hydrolytic degradation of silorane- and methacrylate-based composite restorations: evaluation of push-out strength and marginal adaptation," Acta Odontologica Scandinavica, vol. 71, no. 5, pp. 1273-1279, 2013.

[21] T. R. Aguiar, C. M. P. Vidal, R. S. Phansalkar et al., "Dentin biomodification potential depends on polyphenol source," Journal of Dental Research, vol. 93, no. 4, pp. 417-422, 2014.

[22] C. S. Castellan, A. K. Bedran-Russo, S. Karol, and P. N. R. Pereira, "Long-term stability of dentin matrix following treatment with various natural collagen cross-linkers," Journal of the Mechanical Behavior of Biomedical Materials, vol. 4, no. 7, pp. 1343-1350, 2011.

[23] M. A. Moreira, N. O. Souza, R. S. Sousa et al., "Efficacy of new natural biomodification agents from Anacardiaceae extracts on dentin collagen cross-linking," Dental Materials, vol. 33, no. 10, pp. 1103-1109, 2017.

[24] A. Tezvergil-Mutluay, R. Seseogullari-Dirihan, V. P. Feitosa et al., "Zoledronate and ion-releasing resins impair dentin 
collagen degradation," Journal of Dental Research, vol. 93, no. 10, pp. 999-1004, 2014.

[25] L. Fu, X. Sun, Y. Gao, and R. Chen, "Tannic acid: a novel calibrator for facile and accurate mass measurement of electrospray ionization mass spectrometry," Journal of the American Society for Mass Spectrometry, vol. 30, no. 8, pp. 1545-1549, 2019.

[26] A. K. B. Bedran-Russo, C. S. Castellan, M. S. Shinohara, L. Hassan, and A. Antunes, "Characterization of biomodified dentin matrices for potential preventive and reparative therapies," Acta Biomaterialia, vol. 7, no. 4, pp. 1735-1741, 2011.

[27] C. Parise Gré, D. Pedrollo Lise, A. P. Ayres et al., "Do collagen cross-linkers improve dentin's bonding receptiveness?" Dental Materials, vol. 34, no. 11, pp. 1679-1689, 2018.

[28] M. P. N. Fialho, V. Hass, R. P. Nogueira et al., "Effect of epigallocatechin-3- gallate solutions on bond durability at the adhesive interface in caries-affected dentin," Journal of the Mechanical Behavior of Biomedical Materials, vol. 91, pp. 398-405, 2019.

[29] E. Haslam, "Natural polyphenols (vegetable tannins) as drugs: possible modes of action," Journal of Natural Products, vol. 59, no. 2, pp. 205-215, 1996.

[30] V. Natarajan, N. Krithica, B. Madhan, and P. K. Sehgal, "Preparation and properties of tannic acid cross-linked collagen scaffold and its application in wound healing," Journal of Biomedical Materials Research Part B: Applied Biomaterials, vol. 101B, no. 4, pp. 560-567, 2013.

[31] M. Jastrzebska, J. Zalewska-Rejdak, R. Wrzalik et al., “Tannic acid-stabilized pericardium tissue: IR spectroscopy, atomic force microscopy, and dielectric spectroscopy investigations," Journal of Biomedical Materials Research Part A, vol. 78A, no. 1, pp. 148-156, 2006.

[32] A. K. B. Bedran-Russo, K. J. Yoo, K. C. Ema, and D. H. Pashley, "Mechanical properties of tannic-acid-treated dentin matrix," Journal of Dental Research, vol. 88, no. 9, pp. 807-811, 2009.

[33] S. B. Botta, P. A. Ana, M. O. Santos, D. M. Zezell, and A. B. Matos, "Effect of dental tissue conditioners and matrix metalloproteinase inhibitors on type I collagen microstructure analyzed by Fourier transform infrared spectroscopy," Journal of Biomedical Materials Research Part B: Applied Biomaterials, vol. 100B, no. 4, pp. 1009-1016, 2012.

[34] V. Hass, I. Luque-Martinez, M. A. Muñoz et al., "The effect of proanthocyanidin-containing $10 \%$ phosphoric acid on bonding properties and MMP inhibition," Dental Materials: Official Publication of the Academy of Dental Materials, vol. 32, no. 3, pp. 468-475, 2016.

[35] R.-R. Liu, M. Fang, L. Zhang, C.-F. Tang, Q. Dou, and J.-H. Chen, "Anti-proteolytic capacity and bonding durability of proanthocyanidin-biomodified demineralized dentin matrix," International Journal of Oral Science, vol. 6, no. 3, pp. 168-174, 2014.

[36] D. J. Epasinghe, C. K. Y. Yiu, M. F. Burrow, N. Hiraishi, and F. R. Tay, "The inhibitory effect of proanthocyanidin on soluble and collagen-bound proteases," Journal of Dentistry, vol. 41, no. 9, pp. 832-839, 2013.

[37] J.-W. Nam, R. S. Phansalkar, D. C. Lankin et al., "Subtle chemical shifts explain the NMR fingerprints of oligomeric proanthocyanidins with high dentin biomodification potency," The Journal of Organic Chemistry, vol. 80, no. 15, pp. 7495-7507, 2015.

[38] A. A. Leme, C. M. P. Vidal, L. S. Hassan, and A. K. BedranRusso, "Potential role of surface wettability on the long-term stability of dentin bonds after surface biomodification," Journal of Biomechanics, vol. 48, no. 10, pp. 2067-2071, 2015.

[39] A. Balalaie, M. B. Rezvani, and M. Mohammadi Basir, "Dual function of proanthocyanidins as both MMP inhibitor and crosslinker in dentin biomodification: a literature review," Dental Materials Journal, vol. 37, no. 2, pp. 173-182, 2018.

[40] D. J. Epasinghe, C. K. Y. Yiu, M. F. Burrow, J. K. H. Tsoi, and F. R. Tay, "Effect of flavonoids on the mechanical properties of demineralised dentine," Journal of Dentistry, vol. 42, no. 9, pp. 1178-1184, 2014.

[41] B. Brodsky and A. V. Persikov, "Molecular structure of the collagen triple helix," Fibrous Proteins: Coiled-Coils, Collagen and Elastomers, vol. 70, pp. 301-339, 2005.

[42] A. J. Gwinnett, "Quantitative contribution of resin infiltration/hybridization to dentin bonding," American Journal of Dentistry, vol. 6, no. 1, pp. 7-9, 1993.

[43] L. Zou, L. Zhang, J. Li et al., "Effect of Galla chinensis extract and chemical fractions on demineralization of bovine enamel in vitro," Journal of Dentistry, vol. 36, no. 12, pp. 999-1004, 2008.

[44] T.-T. Zhang, H.-J. Guo, X.-J. Liu, J.-P. Chu, and X.-D. Zhou, "Galla chinensis compounds remineralize enamel caries lesions in a rat model," Caries Research, vol. 50, no. 2, pp. 159-165, 2016.

[45] Z. Khurshid, M. S. Zafar, S. Zohaib, S. Najeeb, and M. Naseem, "Green tea (Camellia sinensis): chemistry and oral health," The Open Dentistry Journal, vol. 10, no. 1, pp. 166-173, 2016. 